ESJ Natural/Life/Medical Sciences

\title{
Étude Ethnobotanique Des Plantes Spontanées Comestibles Dans Le Département De Zuénoula (Centre-Ouest De La Côte D'ivoire)
}

\author{
Vanié-Bi Irié Germain \\ Laboratoire d'Agro-physiologie, UFR Biosciences, \\ Université Félix Houphouët-Boigny, Abidjan, Côte d'Ivoire
}

Béné Kouadio

UFR des Sciences de la Nature (SN), Université Nangui Abrogoua, Abidjan, Côte d'Ivoire

Zouzou Michel

Laboratoire d'Agro-physiologie, UFR Biosciences, Université Félix Houphouët-Boigny, Abidjan, Côte d’Ivoire

Doi:10.19044/esj.2021.v17n29p242

Submitted: 06 February 2021

Accepted: 27 April 2021

Copyright 2021 Author(s)

Published: 31 August 2021

Under Creative Commons BY-NC-ND

Cite As:

Germain V-B.I., Kouadio B. \& Michel Z.(2021). Étude Ethnobotanique Des Plantes Spontanées Comestibles Dans Le Département De Zuénoula (Centre-Ouest De La Côte D'ivoire). European Scientific Journal, ESJ, 17(29), 242.

https://doi.org/10.19044/esj.2021.v17n29p242

\section{Résumé}

Cette étude a été conduite à Zuénoula, dans le but d'évaluer la disponibilité et le niveau de connaissance des plantes sauvages comestibles pouvant servir d'alternatives aux populations locales et contribuer ainsi à leur valorisation. Pour cela, des investigations ethnobotaniques ont été réalisées à l'aide de fiches d'enquête dans 5 localités auprès de 368 individus. Les plantes et les organes comestibles récoltés ont été identifiés au Centre National de Floristique (CNF) d'Abidjan. Ainsi, les 76 espèces récensées appartiennent à 36 familles et 62 genres et représentent 32,34\% des plantes sauvages comestibles recensées en Côte d'Ivoire. Les Fabaceae (33,34 \%) et les Malvaceae $(22,23 \%)$ sont les familles les plus rencontrées et sont majoritairement sous forme d'arbres (38,16 \%) et d'herbes (26,32 \%). Concernant les parties utilisées, ce sont les feuilles (38,85\%) et les graines 
(07,45 \%) qui sont plus consommées en repas contrairement aux fruits $(40,43$ \%) souvent consommés comme friandise au lieu de cueillette. Les populations (62,23 \%) qui ont une bonne connaissance de la flore locale comestible sont aujourd'hui confrontées à la disparition progressive d'espèces autrefois prisées comme Glyphaea brevis, Irvingia gabonensis et Telfairia occidentalis. Enfin, la consommation de Bixa orellana et de Gymnema sylvestre, espèces très utilisées dans l'alimentation humaine et la médecine en Asie, a pour la première fois été révélée en Côte d'Ivoire. La poursuite de cette étude par des analyses des valeurs nutritives des espèces les plus sollicitées est vivement souhaitée en vue de leur valorisation au plan nutritionnel.

Mots-clés: Ethnobotanique, Comestibles, Sauvages, Zuénoula, Côte d’Ivoire

\title{
Ethnobotanical Study Of Spontaneous Edible Plants In The Department Of Zuenoula (Center-West Of Côte D’ivoire)
}

\author{
Vanié-Bi Irié Germain \\ Laboratoire d'Agro-physiologie, UFR Biosciences, \\ Université Félix Houphouët-Boigny, Abidjan, Côte d'Ivoire
}

\section{Béné Kouadio}

UFR des Sciences de la Nature (SN), Université Nangui Abrogoua, Abidjan, Côte d'Ivoire

Zouzou Michel

Laboratoire d'Agro-physiologie, UFR Biosciences, Université Félix Houphouët-Boigny, Abidjan, Côte d’Ivoire

\section{Abstract}

This study was conducted in Zuénoula with the aim of assessing the local people's level of knowledge on wild edible plants and the availability of these plants to serve as alternatives for the local population and thus contribute to their development. To achieve this, botanical surveys were carried out in 5 localities involving 368 persons. Plants and edible organs collected were identified at the Centre National de Floristique (CNF) in Abidjan. All 76 species recorded belong to 36 families and 62 genera, and they represent $32.34 \%$ of wild edible plants identified in Côte d'Ivoire. Fabaceae (33.34\%) and Malvaceae (22.23\%) were the most common families and are mainly trees (38.16\%) and herbs (26.32\%). Concerning the part of the plants used, leaves (38.85\%) and seeds $(07.45 \%)$ were the most consumed in meals, which is contrary to fruits (40.43\%) often consumed as sweets at the harvesting sites. The majority of the population (62.23\%), which has a good knowledge of the local edible flora, is today confronted with the progressive disparition of 
species once sought after such as Glyphaea brevis, Irvingia gabonensis, and Telfairia occidentalis. This study reveals for the first time the consumption in Côte d'Ivoire of two plants' species, Bixa orellana and Gymnema sylvestre, widely used in human food and medicine in Asia, highlighting the great diversity of the local flora. Since the nutritional potential of these species has never been studied in Côte d'Ivoire, additional research on the most soughtafter species is highly recommended for their nutritional valorization.

Keywords: Ethnobotany, Edibles, Wild, Zuénoula, Côte d’Ivoire

\section{Introduction}

Depuis son indépendance (1960), la Côte d'Ivoire s'est dotée d'un modèle de développement agricole exemplaire pour répondre aux objectifs socio-économiques très variés (sécurité alimentaire, accroissement des devises, couverture nationale en produits agricoles locaux, etc.). Les reformes dans ce secteur au fil des années ont permis au pays de batir un développement économique florissant, qualifié de "miracle ivoirien », et de se maintenir parmi les grandes nations agro-productrices mondiales dans plusieurs domaines notamment ceux du cacao, du café, des noix de cajou et de cola, de l’hévéa, de l’igname, du manioc, etc. (Ducroquet et al., 2017).

Malgré ces performances, les populations rurales se trouvent dans des conditions économiques, sociales et environnementales préoccupantes. En effet, avec la hausse vertigineuse de plus de $50 \%$ du niveau de pauvreté en trois décennies, surtout en zones rurales où les conditions de vie des paysans dépendent des activités agricoles (70 à 90 \%) elles-mêmes menacées par les sécheresses, la Côte d'Ivoire fait partie des nations au monde (172ième sur 182) à faibles indices du développement humain (PNUD, 2015). De même, avec plus de $84 \%$ des forêts disparues en moins de cinq décennies (SODEFOR, 1996 ; Koné, 2015), et son manque de préparation efficace face à la sécheresse, la Côte d'Ivoire est classée $147^{\text {ième }}$ sur 178 pays les plus vulnérables aux impacts du réchauffement climatique (Banque mondiale, 2018).

Afin de faciliter durablement l'accès aux aliments en milieu rural, il faut développer de nouvelles cultures vivrières, mieux adaptées aux situations socio-économiques et environnementales des populations locales. Les variétés améliorées à meilleur rendement ayant montré leurs limites dans les agrosystèmes paysans (cultures exigeantes, manque de rusticités), il apparait opportun de valoriser les plantes indigènes, abandonnées dans la nature, alors qu'elles disposent d'un patrimoine productif rustique et d'un potentiel alimentaire sain.

En effet, en Afrique tropicale et particulièrement en Côte d'Ivoire, les plantes sauvages comestibles possèdent de nombreux avantages aux plans 
économique, alimentaire, social, thérapeutique, cosmétique et industriel (N'Guessan et al., 2015). C'est le cas des ignames sauvages du genre Dioscorea dont les espèces $D$. praehensilis, $D$. odoratissima et $D$. smilacifolia, furent utilisées comme aliments énergétiques de base durant des saisons sèches dans certaines régions de Côte d'Ivoire (Ouattara et al., 2016). Aujourd'hui, de nombreuses espèces jouent une double fonction (Nutrition/Médecine). Parmi elles, Solanum indicum et Solanum nigrum sont utilisées comme nourritures et remontants aux malades en cas de fatigue générale. Ces végétaux sont des sources de micronutriments essentiels comme le fer, le calcium, le zinc, la vitamine A, dont les carences constituent des problèmes recurrents de santé publique (N’Guessan et al., 2015). Au plan de l'autosubsistance financière des femmes rurales, les amandes de Irvingia gabonensis par exemple, utilisées pour confectionner des sauces prisées en milieu rural, sont également vendues à 3000 francs le kilogramme (6 dollars) dans les marchés locaux, largement supérieurs aux prix actuels de fèves de cacao (2 dollars), de grains de café, de palmier, de noix d'acajou (tous moins de 1 dollar). De ce fait, ces amendes font l'objet d'un commerce florissant.

Ainsi, la valorisation des végétaux sauvages comestibles, intégrant le patrimoine socio-économique et environnemental des paysans apparait comme une stratégie alternative pour accroitre durablement les revenus et améliorer les repas des ménages pauvres en zone rurale. C'est dans ce cadre que cette étude a été menée sur les plantes spontanées comestibles qui poussent dans la Région de la Marahoué, plus particulièrement dans le Département de Zuénoula.

La Région Marahoué est reconnue comme une zone de production vivrière, activité principale des femmes rurales. Mais, dans certains Départements comme celui de Zuénoula, seuls les besoins énergétiques de base des populations sont satisfaits, et ceci pendant les périodes de récoltes des principaux aliments (plantains, ignames, riz, manioc). De plus, les forêts et les jachères, autrefois réservées aux cultures vivrières, se sont considérablement réduites, et la population est devenue plus pauvre avec près de $62 \%$ de pauvreté dans les villages (Ducroquet et al., 2017). Toutefois, certains ménages parmi les plus démunis continuent d'exploiter les espèces sauvages pour satisfaire à leurs besoins, soit par des cueillettes en brousses, soit par la création de modestes potagers familiaux (HLPE, 2018). Afin d'appuyer ces ménages et étendre leurs initiatives à l'ensemble des communautés locales, il faut au préalable disposer d'un répertoire de plantes sauvages comestibles encore présentes dans la flore du Département, facilitant le choix des variétés à valoriser. Malheureusement, aucun registre d'espèces sauvages comestibles à Zuénoula n'est établi. Le Professeur Aké Assi et son équipe, ainsi que d'autres chercheurs, ont sans doute exploré la Région, mais aucun des auteurs n'en parle, alors que d'autres régions de la Côte d'Ivoire 
disposent déjà de cet outil scientifique: Divo (N'Dri, 1986), Toumodi (Gautier-Beguin, 1992), Oumé (Kouamé, 2000), Séguéla (Ambé, 2003), Gagnoa (Kouamé et al., 2008), Agboville (N’guessan, 2015), Bondoukou (Ouattara et al., 2016), etc. La seule enquête botanique récente effectuée dans le Département fut le recensement des plantes médicinales antidiabétiques utilisées par les populations locales (Gnagne et al., 2017).

Ce travail est une réponse à cette préoccupation. L'étude ainsi menée a pour objectif la connaissance de la diversité des espèces sauvages comestibles présentes dans la flore spontanée du Département de Zuénoula.

\section{Matériel Et Méthodes \\ Zone d'étude}

L’étude a été conduite dans le Département de Zuénoula. Celui-ci fait partie de la Région de la Marahoué située au Centre-Ouest de la Côte d'Ivoire, entre les latitudes $7^{\circ} 10^{\prime} 50^{\prime \prime}$ et $7^{\circ} 45^{\prime} 10^{\prime \prime} \mathrm{N}$ et les longitudes $5^{\circ} 43^{\prime} 12^{\prime \prime}$ et $6^{\circ} 26^{\prime} 40^{\prime \prime} \mathrm{W}$. Le Département comprend une Préfecture et sept SousPréfectures, la ville de Zuénoula étant le Chef-lieu de Département (Figure 1). Il est composé de 112 villages et plus de 1048 campements. Il s'étend sur une superficie de $3252 \mathrm{~km}^{2}$. Sa population est passée de 172191 habitants en 2004 à 214646 en 2014 (RGPH, 2014). Plus de $65 \%$ de cette population est rurale et vit principalement de l'agriculture (Ducroquet et al., 2017). En plus des Gouro, peuple autochtone majoritaire, la Région abrite des communautés ivoiriennes et une forte concentration des ressortissants de la sous-région.

Le Département de Zuénoula est une zone de transition savanes-forêts ou zone soudano-guinéenne (PDI, 2003). La végétation est constituée de mosaïque de forêts et de savanes boisées au Nord, et de forêt dense humide semi-décidue au Sud-Ouest, fortement dégradée par les activités humaines (N'Da et al., 2008). Les sols sont de types ferralitiques, laissant apparaitre au Sud-Ouest, de collines et des affleurements granitiques. Le climat est du type tropical avec une température moyenne annuelle de $29^{\circ} \mathrm{C}$. La pluviométrie moyenne annuelle est passée de $1800 \mathrm{~mm}$ en 1980 à $1200 \mathrm{~mm}$ en 2013 (Yao et al., 2013). Un seul cours d'eau permanent traverse du Nord au Sud le Département : c’est le Bandaman rouge ou la Marahoué.

Dans ce travail, les enquêtes ont été réalisées dans cinq SousPréfectures, choisies en fonction de leurs positions géographiques, et dans des localités où l'occupation humaine est relativement dense : Gohitafla, Kanzra, Vouéboufla, Zanzra, Zuénoula.

\section{Matériel}

\section{Matériel biologique}

Il est constitué des plantes comestibles poussant à l'état sauvage reconnues par les populations locales. 


\section{Matériel technique}

C'est l'ensemble des outils classiques de récolte et de mesures en Botanique à savoir des sécateurs pour prélever les organes, des appareils numériques de prises de vues et de positions géographiques pour capter les images et localiser les sites de prélèvement, des fiches cartonnées, de journaux pour la confection des herbiers.

\section{Méthodes}

\section{Choix des personnes retenues pour les enquêtes}

Les enquêtes ont été réalisées auprès de trois communautés villageoises : les Autochtones (les Gouro ), les Allogènes (les Baoulé et les Senoufo), et les Burkinabé (peuples immigrés). Pour le recensement et la collecte des espèces, des ressortissants ayant une bonne connaissance des plantes ont été choisis de façon aléatoire dans les cinq SousPréfectures, après une pré-enquête ayant permis de les identifier. Par la suite, les niveaux de connaissance et de consommation des plantes ont été évalués uniquement auprès des populations Gouro, peuples majoritaires du Département. Pour toutes ces enquêtes, la méthode semi-structurée a été utilisée pour recueillir des informations, à l'aide de la fiche d'enquête.

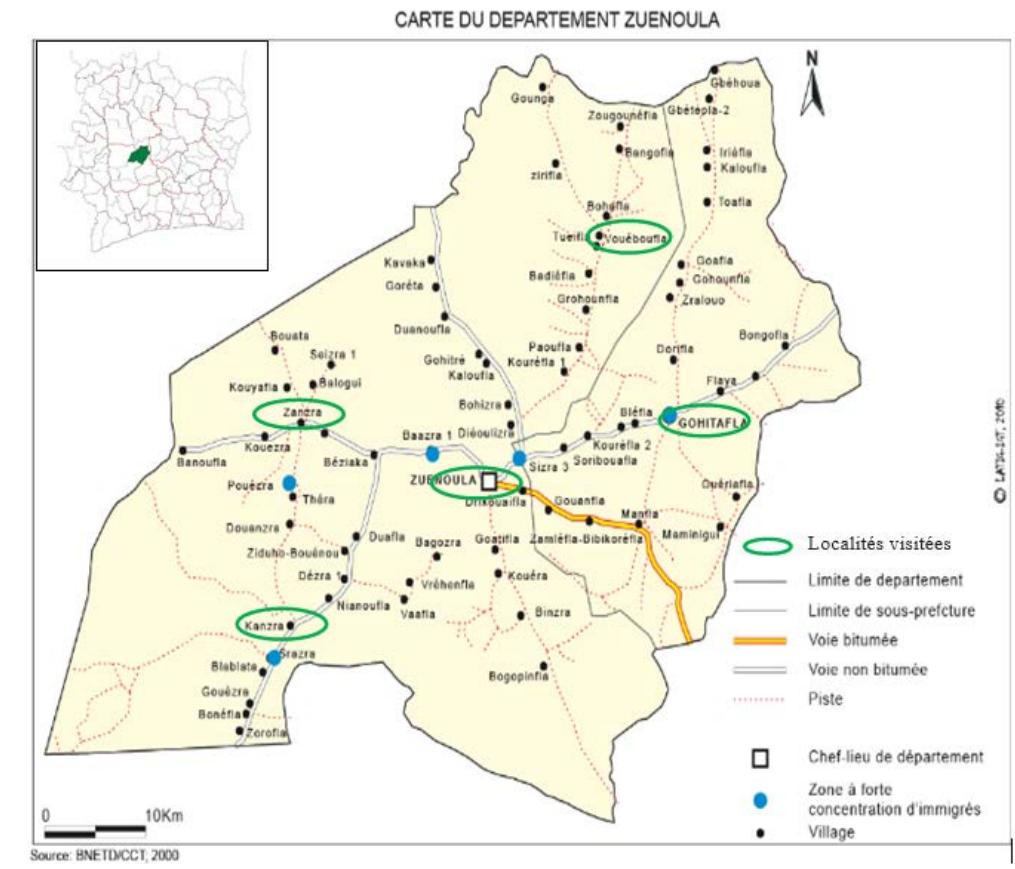

Figure 1. Carte du Département de Zuénoula situant les localités enquêtées 


\section{Enquête ethnobotanique}

L'enquête a débuté le 02 avril 2018 puis s'est achevée le 11 août 2019. Elle s'est déroulée aussi bien pendant les saisons sèches que les saisons pluvieuses. Les sorties botaniques ont consisté en des incursions dans des savanes, des forêts, des plantations et des jachères avec les guides choisis, comme dans des précédentes études botaniques (Kouamé et al, 2008; Ouattara et al., 2016). Les plantes comestibles reconnues ont été recensées et des échantillons ont été collectés. Pour chaque espèce, le nom en langue locale Gouro a été mentionné, et quelque fois en langue Moré (peuple du Burkina Faso). A la fin de chaque mission, les herbiers ont été constitués puis déposés au Centre National de Floristique d'Abidjan, en vue d'identifier les espèces. Cette identification s'est déroulée par la reconnaissance spontanée de l'espèce, et parfois par comparaison aux spécimens déjà décrits dans la flore de Côte d'Ivoire et des ouvrages botaniques notamment ceux de Aké-Assi et de Adjanohoun (Adjanohoun et Aké-Assi, 1979 ; Aké-Assi, 1984, 2001). Par la suite, la nomenclature a été actualisée selon la version III de la classification phylogénétique des Angiospermes (APG IV, 2016).

\section{Évaluation des niveaux de connaissances et de consommation}

A l'aide d'une fiche d'enquête, plusieurs questions ont été posées sur l'usage alimentaire des plantes. Trois d'entre elles ont été nécessaires pour cette rubrique : (1) connaissez-vous la plante, (2) la connaissez-vous comme une plante comestible, (3) l'avez-vous une fois consommée. La question (1) a permis d'évaluer le niveau de connaissance spontanée de l'espèce, tandis que la question (2) a été en rapport à sa comestibilité. Les réponses à la question (3) ont permis de déterminer le niveau de consommation de l'espèce concernée. Ces deux niveaux ont été calculés selon la méthode de Dajoz (Dajoz, 1982) comme suit :

où

$$
P=\frac{n}{N} 100
$$

$\boldsymbol{P}$ désigne le niveau de connaissance de l'espèce (ou de consommation), $\boldsymbol{n}$ désigne le nombre de personnes ayant reconnue l'espèce (ou consommé une fois), $\boldsymbol{N}$ désigne le nombre total de personnes interrogées. Selon cette méthode, les espèces ont été réparties en trois groupes en fonction des valeurs de $\boldsymbol{P}$. Ainsi, pour $\boldsymbol{P}$ compris entre 50 et $100 \%$, l'espèce est considérée comme plus connue ; pour $\boldsymbol{P}$ compris entre 25 à $50 \%$, l'espèce est considérée moyennement connue; pour $\boldsymbol{P}$ inférieur à $25 \%$, l'espèce est considérée peu connue par les personnes interrogées. Le même procédé a permis de déterminer le niveau de consommation des plantes. 


\section{Analyses statistiques}

Les traitements statistiques ont consisté à déterminer les familles, les espèces, les formes, les organes les plus cités au niveau de la diversité floristique d'une part, et à identifier les catégories sociales les plus sollicitées au niveau des communautés villageoises d'autre part. Pour cela, les résultats des inventaires floristiques, les niveaux de préférence des organes et les communautés ont été soumis au test de Chi 2 pour vérifier si les différences observées dans une série de valeurs ne sont pas significatives au seuil de $5 \%$. Dans le cas contraire, le Chi 2 a été complété par le test de normalité pour comparer les effectifs de la série entre eux.

\section{Résultats}

\section{Profil des personnes enquêtées}

Les enquêtes ont été réalisées auprès de 368 personnes issues des cinq localités administratives du Département (Figure 1). Les Gouro ont représenté $75 \%$ de communautés interrogées contre $25 \%$ des allochtones et des Burkinabé (Figure 2A). Les femmes ont été les plus sollicitées (Figure 2B), de même que les personnes d'âges compris entre 11 et 50 ans (Figure 2C). Dans l'ensemble, la moitié des personnes enquêtées n'a que le niveau d'étude de l’école primaire (Figure 2D).

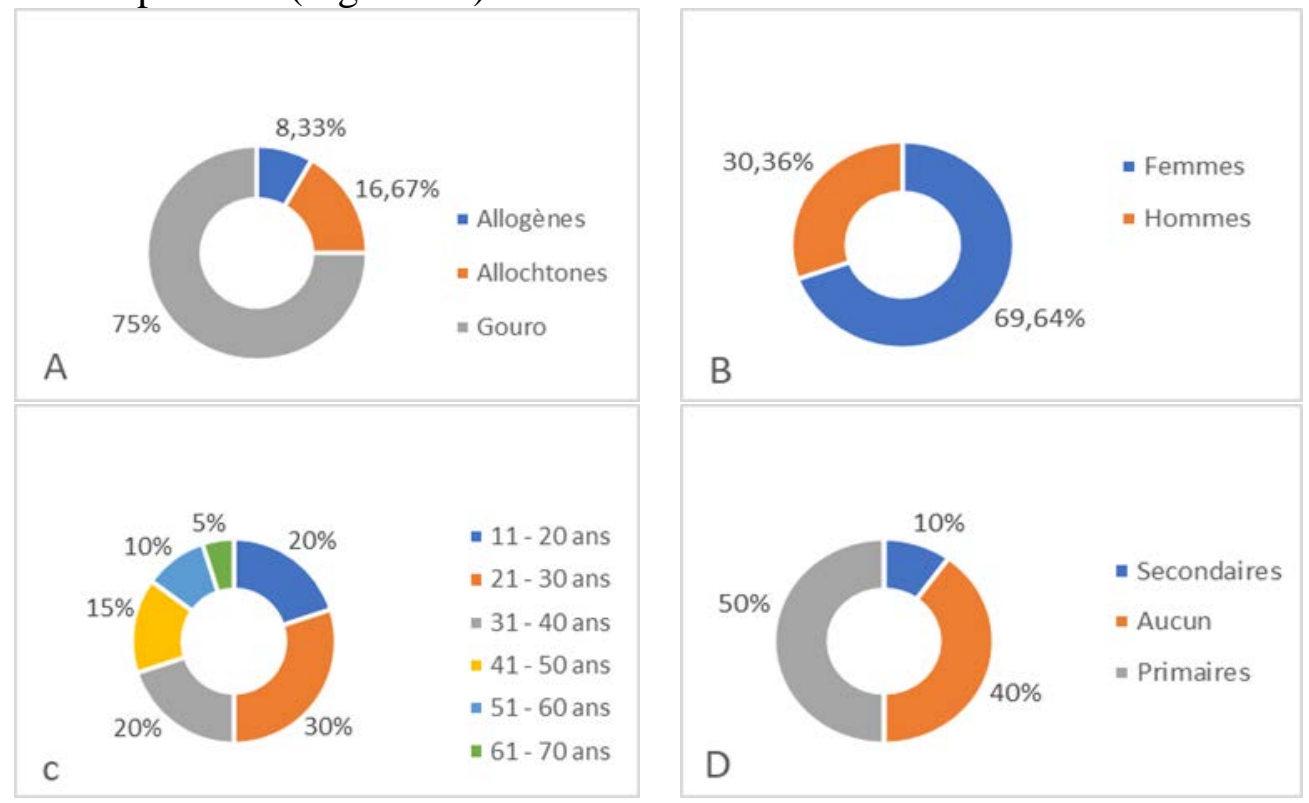

Figure 2. Spectres des catégories sociales et démographiques des interviewés : (A) origine,

(B) genre, (C) âge, (D) niveau d'instruction

\section{Diversité floristique}

Au total, soixante-seize (76) espèces ont été inventoriées dans le Département de Zuénoula (Tableau 1). Les types morphologiques 
apparemment majoritaires sont les arbres (38,16 \%) suivis des herbes (26,32 $\%)$, des arbustes $(21,05 \%)$ et des lianes $(14,47 \%)$. Cette distribution est homogène, le test Chi $2(2,12)$ étant non significatif au seuil de $5 \%$. Ces 76 espèces se répartissent en 62 genres et 36 familles. Les Fabaceae ( 9 espèces) et les Malvaceae (8 espèces) ont été plus recensées (Figure 3). Parmi les espèces inventoriées, une n’a jamais été citée par les auteurs en Côte d'ioire : Gymnema sylvestre.

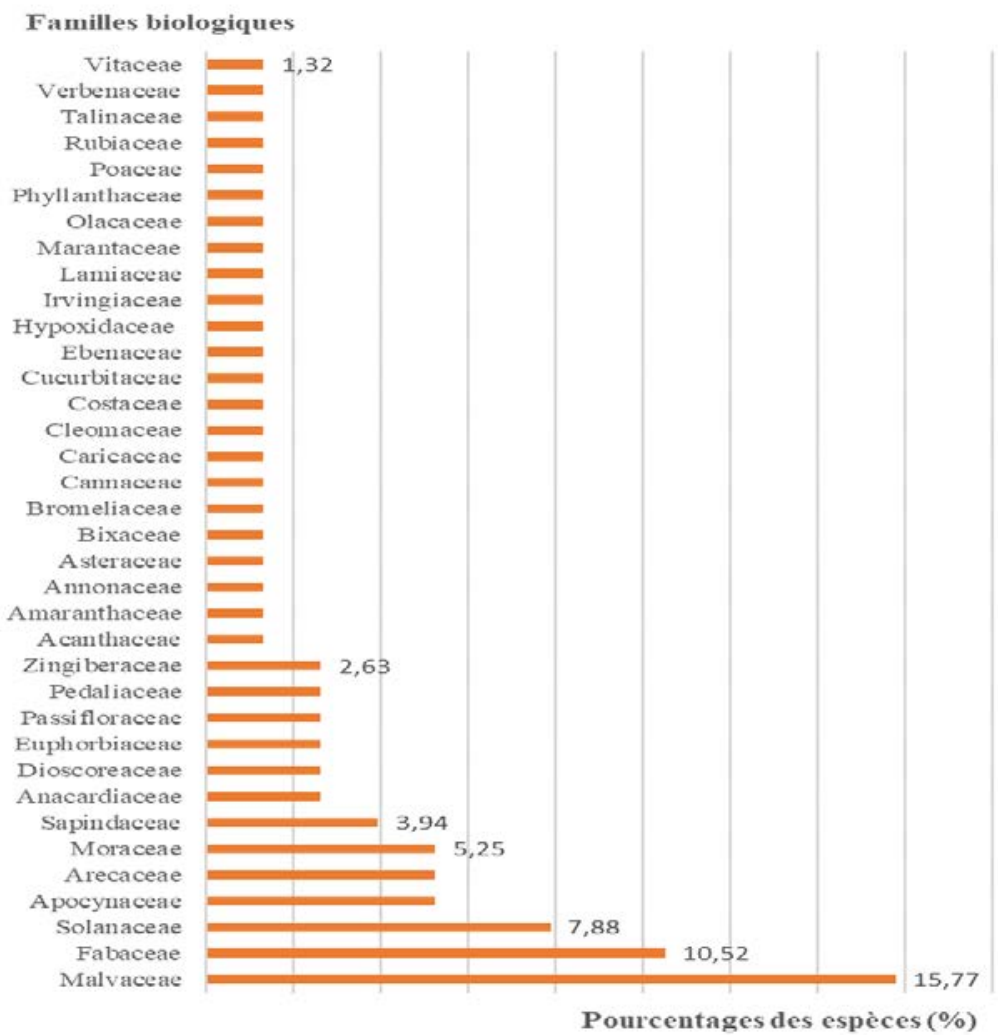

Figure 3. Histogramme des familles en fonction des pourcentages des espèces

Tableau 1. Liste des plantes comestibles sauvages recensées dans le Département de Zuénoula

\begin{tabular}{|l|l|l|l|}
\hline Noms des espèces & Familles & $\begin{array}{l}\text { Formes } \\
\text { biologiques }\end{array}$ & Habitats \\
\hline Abrus precatorius L. & Fabaceae & Liane & Forêts \\
\hline Acalypha ciliata Forssk. & Euphorbiaceae & Herbe & Jachères \\
\hline Adansonia digitata L. & Malvaceae & Arbre & Savanes \\
\hline Adenia cissampeloides (Hook.) Harms & Passifloraceae & Liane & Savanes arborées \\
\hline Aframomum alboviolaceum (Ridl.) K. Schum. & Zingiberaceae & Herbe & Savanes arborées \\
\hline Aframomum melegueta K. Schum. & Zingiberaceae & Herbe & Savanes arborées \\
\hline Albizia zygia (OC.) J. F Macbr & Fabaceae & Arbre & Savanes et forêts \\
\hline Ananas comosus (L.) Merr. & Bromeliaceae & Herbe & Forêts \\
\hline Annona senegalensis Pers. & Annonaceae & Arbuste & Savanes \\
\hline
\end{tabular}




\begin{tabular}{|c|c|c|c|}
\hline Bixa orellana L. & Bixaceae & Arbuste & Forêts \\
\hline Blighia sapida K.D. Koenig & Sapindaceae & Arbre & Forêts \\
\hline Bombax buonopozense P. Beauv & Malvaceae & Arbre & Savanes arborées et forêts \\
\hline Bombax costatum Pellegr. \& Vuillet & Malvaceae & Arbre & Savanes boisées et forêts \\
\hline Borassus aethiopum Mart. & Arecaceae & Arbre & Savanes \\
\hline Canna indica L. & Cannaceae & Herbe & Zones humides \\
\hline Capsicum frutescens L. & Solanaceae & Arbuste & forêts \\
\hline Carica papaya L. & Caricaceae & Arbuste & Forêts \\
\hline Ceiba pentandra (L.) Gaertn. & Malvaceae & Arbre & Forêts \\
\hline Celosia trigyna L. & Amaranthaceae & Herbe & Jachères \\
\hline Cissus populnea Guill. \& Perr. & Vitaceae & Liane & Savanes arborées \\
\hline Cola caricaefolia (G. Don) Schum, & Malvaceae & Arbre & Lisières des forêts \\
\hline Cola gigantea var.glabrescens Brenan et Keay & Malvaceae & Arbre & Savanes \\
\hline Corchorus aestuans L. & Malvaceae & Herbe & Jachères en forêts \\
\hline Corchorus olitorus L. & Malvaceae & Herbe & Jachères en forêts \\
\hline Corchorus tridens L. & Malvaceae & Herbe & Jachères en forêts \\
\hline Costus afer Ker-Gawl. & Costaceae & Herbe & Zones humides des forêts \\
\hline $\begin{array}{l}\begin{array}{l}\text { Curculigo pilosa (Schum. \& Thonn. Engler } \\
\text { (velu). }\end{array} \\
\text { (velu }\end{array}$ & Hypoxidaceae & Herbe & Jachères et forêts \\
\hline Deinbollia pinnata (Poir.) Schum. \& Thonn. & Sapindaceae & Arbuste & Forêts \\
\hline Detarium senegalense J. F.Gmel. & Fabaceae & Arbuste & Savanes arborées \\
\hline Dialium guineense Willd. & Fabaceae & Arbre & Forêts \\
\hline Dioscorea odoratissima Pax & Dioscoreaceae & Liane & Forêts \\
\hline Dioscorea praehensilis Benth. & Dioscoreaceae & Liane & Forêts \\
\hline Diospyros mespiliformis A. OC. & Ebenaceae & Arbre & Forêts \\
\hline Elaeis guineensis Jacq. & Arecaceae & Arbre & Forêts, champs et jachères \\
\hline Ficus exasperata M. Vahl & Moraceae & Arbre & Savanes arborées et forêts \\
\hline Ficus sur Forsk. & Moraceae & Arbre & Savanes arborées \\
\hline Ficus vallis-choudae Delile & Moraceae & Arbre & Savanes arborées \\
\hline Gardenia ternifolia Schum. \& Thonn. & Rubiaceae & Arbuste & Savanes arborées \\
\hline Glyphaea brevis (Spreng.) Monach. & Malvaceae & Arbuste & Forêts et jachères \\
\hline Gymnema sylvestre (Retz) Schult & Apocynaceae & Liane & Savanes arborées \\
\hline Gynandropsis gynandra (L.) Briq. & Cleomaceae & Herbe & Jachères \\
\hline Imperata cylindrica L. Raeuschel & Poaceae & Herbe & Savanes \\
\hline Irvingia gabonensis (O'Rorke)Baill & Irvingiaceae & Arbre & Forêts \\
\hline Justicia galeopsis T. Anderson Ex C.B. Clarke & Acanthaceae & Herbe & Jachères \\
\hline Landolphia heudelotii A. DC. & Apocynaceae & Liane & Forêts \\
\hline Lannea barteri (L. kerstingii) & Anacardiaceae & Arbuste & Savanes arborées \\
\hline Lippia multiflora Moldenke & Verbenaceae & Arbuste & Savane \\
\hline $\begin{array}{l}\text { Lonchocarpus cyanescens (Schum. \& Thonn.) } \\
\text { Ben th. }\end{array}$ & Fabaceae & Arbuste & Savanes arborées et forêts \\
\hline Myrianthus arboreus P. Beauv & Moraceae & Arbre & Forêts \\
\hline Parkia biglobosa (Jacq.) R.Br. ex G. Don & Fabaceae & Arbre & Savanes \\
\hline Passiflora foetida L. & Passifloraceae & Liane & Savanes et jachères \\
\hline Paullinia pinnata $\mathrm{L}$. & Sapindaceae & Liane & Forêts \\
\hline Phoenix reclinata Jacq. & Arecaceae & Arbre & Zones humides \\
\hline Phyllanthus reticulatus Poir. & Phyllanthaceae & Arbuste & Forêts \\
\hline Raphia hookeri Mann. \& Wendl. & Arecaceae & Arbre & Zones humides \\
\hline
\end{tabular}




\begin{tabular}{|l|l|l|l|}
\hline Ricinodendron heudelotii (Baill.) Pierre ex Pax & Euphorbiaceae & Arbre & Forêts \\
\hline Saba comorensis (Bojer) Pichon & Apocynaceae & Liane & Forêts \\
\hline Saba senegalensis (A. DC.) Pichon & Apocynaceae & Liane & Forêts \\
\hline Sesamum indicum L. & Pedaliaceae & Herbe & Savanes et jachères \\
\hline Sesamum radiatum Schum. \& Thonn. & Pedaliaceae & Herbe & Savanes et jachères \\
\hline Solanum americanum Mill. & Solanaceae & Herbe & Plantations et jachères \\
\hline Solanum indicum L. & Solanaceae & Arbuste & Forêts et jachères \\
\hline Solanum nigrum L. & Solanaceae & Herbe & Plantations et jachères \\
\hline Solanum rigosumL. & Solanaceae & Arbuste & Forêts et jachères \\
\hline Solanum torvum Sw. & Solanaceae & Arbuste & Forêts et jachères \\
\hline Spondias mombin L. & Anacardiaceae & Arbre & Savanes arborées \\
\hline Sterculia tragacantha Lindl. & Malvaceae & Arbre & Forêts \\
\hline Talinum triangulare (Jacq.) Willd. & Talinaceae & Herbe & Jachères \\
\hline Tamarindus indica L. & Fabaceae & Arbre & Forêts \\
\hline Telfairia occidentalis Hook. f. & Cucurbitaceae & Liane & Forêts, jachères \\
\hline $\begin{array}{l}\text { Thaumatococcus daniellii (Bennet) Benth. \& } \\
\text { Hook. }\end{array}$ & Marantaceae & Herbe & Forêts, zones humides \\
\hline Triplochiton scleroxylon Schum. & & & \\
\hline Uraria picta (Jacq.) DC. & Malvaceae & Arbre & Forêts \\
\hline Vernonia amygdalina Del. & Fabaceae & Arbuste & Savanes arborées \\
\hline Vitex doniana Sweet & Asteraceae & Arbre & Savanes arborées, forêts \\
\hline Ximenia americana L. & Lamiaceae & Arbre & Savanes arborées \\
\hline
\end{tabular}

\section{Diversité des organes consommés}

Il a été recensé neuf types d’organes réellement consommés : fruits, feuilles, sèves, graines, fleurs, bourgeons végétatifs, rhizomes, nectars (Figure 4). Les fruits (43,75 \%) et les feuilles (29,17\%) ont été les plus cités. La consommation d'un organe particulier, le nectar, a été observée chez une seule espèce, Canna indica. Le nombre d'organes produits par espèce varie de 1 à 4. Le palmier (Elaeis guineensis) est le taxon ayant fourni le plus d'organes comestibles avec quatre produits : la sève, le bourgeon, la graine et le fruit. A la suite, viennent Glyphaea brevis (fleur, fruit, feuille) et Borassus aethiopum (pulpe, bourgeon, graine) avec trois produits (Tableau 2).

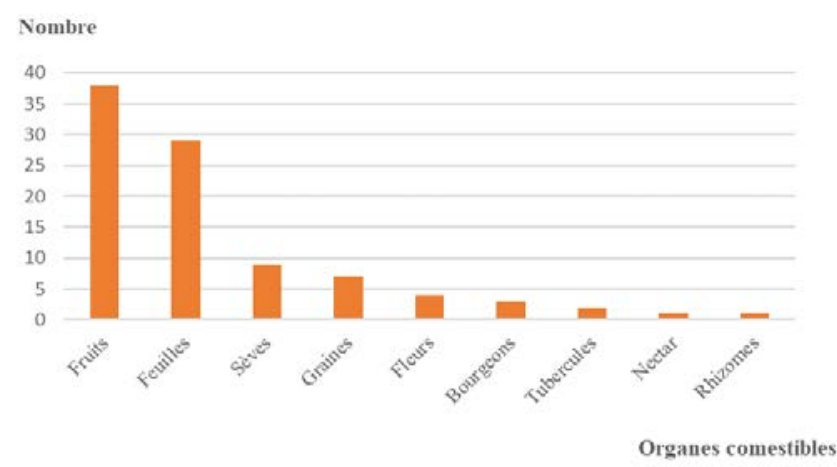

Figure 4. Histogramme des organes consommés 


\section{Niveaux de connaissance et de consommation des espèces}

Les indices de consommations et de connaissances ont été évalués auprès de 200 personnes. Les 76 espèces ont été réparties en trois groupes : 54 espèces ont été bien connues par la population, contre 10 moyennement connues et 12 peu connues. Quant à l'usage de ces plantes, on remarque que 38 espèces ont été déjà consommées par un très grand nombre de personnes (plus de $50 \%$ ), contre 27 espèces peu consommées. Les modes de préparation varient suivant les organes. Ainsi, les feuilles sont bouillies ou cuites avant d'être consommées sous forme d'épinards ou utilisées comme condiments de sauces, contrairement aux fruits qui sont à 92,10 \% consommés crus comme friandises. Les niveaux de connaissance et de consommation des plantes ainsi que les noms des espèces en langues locales Gouro et Moré (Burkina Faso) sont consignés dans le Tableau 2.

Tableau 2. Liste des espèces classées selon leurs indices de connaissance et de consommation des parties comestibles

\begin{tabular}{|c|c|c|c|}
\hline Noms des espèces & $\begin{array}{l}\text { Indices de } \\
\text { connaissance } \\
(\%)\end{array}$ & $\begin{array}{l}\text { Parties comestibles avec } \\
\text { indices de consommation }\end{array}$ & $\begin{array}{l}\text { Noms en langues } \\
\text { locales Gouro et } \\
\text { Moré* }\end{array}$ \\
\hline Adansonia digitata & 100 & Feuilles (92,5 \%), fruits (12 \%) & Bléh \\
\hline $\begin{array}{l}\text { Aframomum } \\
\text { alboviolaceum }\end{array}$ & 100 & Fruits (95 \%) & Srônhon/Zrônhon \\
\hline Ananas comosus & 100 & Fruits $(100 \%)$ & Môlèhè \\
\hline Annona senegalensis & 100 & Fruits (100 \%), feuilles (20 \%) & Blè \\
\hline Blighia sapida & 100 & Fruits (49 \%) & Tchaha/Tchiaha \\
\hline Borassus aethiopum & 100 & $\begin{array}{l}\text { Fruits (23 \%), sève (19,5 \%), } \\
\text { bourgeons ( } 8 \%)\end{array}$ & $Z \grave{e}$ \\
\hline Canna indica & 100 & $\operatorname{Nectar}(76,5 \%)$ & Tolo vohin \\
\hline Carica papaya & 100 & Fruits (100\%) & Vinh \\
\hline Celosia trigyna & 100 & Feuilles $(97,5 \%)$ & Pala yrôlô/Yrôlô \\
\hline Corchorus olitorus & 100 & Feuilles (100 \%) & Miahanin/Mihanin \\
\hline Deinbollia pinnata & 100 & Fruits $(94,5 \%)$ & Palé lé lopo \\
\hline Elaeis guineensis & 100 & $\begin{array}{l}\text { Fruits (100 \%), graines (100 } \\
\%) \text {, } \\
\text { sève (100 \%), bourgeons (5 \%) }\end{array}$ & Yôh \\
\hline Imperata cylindrica & 100 & Rhizomes (33,5 \%) & Lôhlô \\
\hline Irvingia gabonensis & 100 & Graines (100 \%) & Kakrou \\
\hline Lippia multiflora & 100 & Feuilles (94,5 \%) & Fonfon \\
\hline Parkia biglobosa & 100 & Fruits (100\%), graines (18\%) & Minnin minlin \\
\hline Passiflora foetida & 100 & Fruits (100 \%) & Minnin lé sronhon \\
\hline Paullinia pinnata & 100 & Fruits (94 \%) & Bôhô le goii \\
\hline Ricinodendron heudelotii & 100 & Fruits $(100 \%)$ & Kôhô \\
\hline Sesamum radiatum & 100 & Feuilles (100\%) & Nihi \\
\hline Solanum americanum & 100 & Feuilles (100 \%) & Vloun \\
\hline Solanum indicum & 100 & Fruits $(100 \%)$ & Plni \\
\hline Solanum nigrum & 100 & Feuilles (100\%) & Nounou \\
\hline Spondias mombin & 100 & Fruits (100\%), feuilles (46 \%) & Winh \\
\hline
\end{tabular}




\begin{tabular}{|c|c|c|c|}
\hline Sterculia tragacantha & 100 & Feuilles (81,5 \%) & Touhn wini \\
\hline Thaumatococcus daniellii & 100 & Fruits (43,5\%) & Blawèlè \\
\hline Vitex doniana & 100 & Fruits (92 \%) & Plouh \\
\hline Ceiba pentandra & 99,5 & Feuilles (26\%) & Gbin \\
\hline Talinum triangulare & 99,5 & Feuilles (58 \%) & Nango brou \\
\hline Solanum rigosum & 99 & Fruits (2 \%) & Ba Kohou \\
\hline Landolphia heudelotii & 98 & Fruits (95 \%), sève (62 \%) & Toh nin \\
\hline Saba comorensis & 98 & Fruits $(95,5 \%)$, sève $(62 \%)$ & Bô toh nin \\
\hline Myrianthus arboreus & 97,5 & Feuilles (90 \%) & Doha \\
\hline Phoenix reclinata & 97 & Sève (5 \%), fruits (12 \%) & Duanhan ninh \\
\hline Cola gigantea & 95,5 & Fruits (2 \%), feuilles (2\%) & Voho \\
\hline Saba senegalensis & 95 & Fruits (95 \%), sève (21 \%) & Toh nin \\
\hline Gynandropsis gynandra & 93,5 & Feuilles (91,5 \%) & Sanwôh \\
\hline Capsicum frutescens & 92,5 & Fruits $(92,5 \%)$ & Déclé wihnin \\
\hline Ficus vallis-choudae & 91,5 & Fruits (14,5\%) & Sah ylé \\
\hline Bombax buonopozense & 90 & Feuilles (36 \%) & Vlè gbin \\
\hline Dioscorea praehensilis & 88 & Tubercules $(63,5 \%)$ & Sèssè \\
\hline Bombax costatum & 87,5 & Fleurs $(32,5 \%)$ & Gbin \\
\hline Ficus exasperata & 87,5 & Feuilles $(4,5 \%)$ & Tchan non \\
\hline Albizia zygia & 86 & Feuilles (86 \%) & Drounh/Nôhin \\
\hline Cissus populnea & 85,5 & Fruits (85 \%), fleurs (21\%) & Blôhou \\
\hline Ficus sur & 84 & Fruits (7,5 \%), feuilles (7,5 \%) & Môèhnin \\
\hline Dialium guineense & 83,5 & Fruits (56\%) & Banh nin/Blèti \\
\hline Bixa orellana & 76,5 & Graines (63,5 \%) & Po tran po \\
\hline Dioscorea odoratissima & 76,5 & Tubercules (26,5 \%) & Zèh nin \\
\hline Raphia hookeri & 76 & $\begin{array}{l}\text { Sève }(72,5 \%) \text {, bourgeons (47 } \\
\%)\end{array}$ & Bloh/Baha \\
\hline Costus afer & 72,5 & Sève (55 \%) & Zohou \\
\hline Cola caricaefolia & 62 & Fruits (59,5\%) & Glouèglou \\
\hline Acalypha ciliata & 58 & Feuilles (35\%) & Sôla sônin \\
\hline Sesamum indicum & 55 & Graines ( 51,5 \%) & Douwlè \\
\hline Justicia galeopsis & 49 & Feuilles (38\%) & Monhon \\
\hline Phyllanthus reticulatus & 48 & Feuilles (31,5 \%) & Moï nin wih guih'n \\
\hline Triplochiton scleroxylon & 48 & Feuilles (38 \%) & Duhè \\
\hline Curculigo pilosa & 47,5 & Fruits (40,5\%) & Cli lé lo \\
\hline Abrus precatorius & 43 & Feuilles (43\%) & Tchili wlè \\
\hline Glyphaea brevis & 42,5 & $\begin{array}{l}\text { Fruits }(40 \%) \text {, feuilles (27 \%), } \\
\text { fleurs }(14 \%)\end{array}$ & Kayamoè \\
\hline Aframomum melegueta & 42 & Graines (28\%) & Suhè \\
\hline Uraria picta & 41,5 & Fleurs (28 \%) & Cli lé wè \\
\hline Vernonia amygdalina & 39,5 & Feuilles $(12,25) \%$ & Niein niein \\
\hline Adenia cissampeloides & 29,5 & Sève (23,5 ù) & Yi toh mèh \\
\hline Diospyros mespiliformis & 26 & Fruits (19\%) & Garka* \\
\hline Tamarindus indica & 23,5 & Fruits (23,5\%) & Tomi \\
\hline Corchorus aestuans & 22 & Feuilles (2,5\%) & Blin-ninnin \\
\hline Lonchocarpus cyanescens & 21,5 & Feuilles (10,5 \%) & Kangarga* \\
\hline Telfairia occidentalis & 18,5 & Graines $(10,5 \%)$ & Bounin \\
\hline Corchorus tridens & 16 & Feuilles (3,5\%) & Blin-truin \\
\hline
\end{tabular}




\begin{tabular}{|l|l|l|l|}
\hline Detarium senegalense & 9 & Fruits (3\%) & Karakga* $^{*}$ \\
\hline Gardenia ternifolia & 9 & Fruits (3,5\%) & Monnin yli \\
\hline Gymnema sylvestre & 7,5 & Feuilles (7\%) & Lehénougnounou* \\
\hline Solanum torvum & 6 & Fruits $(3,5 \%)$ & Dri plni \\
\hline Lannea barteri & 2,5 & Fruits $(2,5 \%)$ & Samtourouga* \\
\hline Ximenia americana & 2 & Fruits $(1,5 \%)$ & Wanwan yli \\
\hline
\end{tabular}

Indices $<25 \%$ : espèces peu connues/organes peu consommés ; $25 \%<$ Indices $<50 \%$ :

espèces moyennement connues/organes moyennement consommés ; Indices $>50 \%$ : espèces très connues/organes très consommés

\section{Discussion}

La répartition $75 \%$ Gouro contre $25 \%$ allogènes indique que le brassage interethnique est bien prononcé dans le Département de Zuénoula. En effet, à la faveur de la décennie de crises militaro-politiques qu'a connue la Côte d'Ivoire (2002 à 2011), et qui avaient coupé le Département de Zuénoula en deux, la partie Nord étant dans la zone d'exclusion gouvernementale, de nombreux ressortissants de la Sous-Région et des nationaux se sont massivement installés dans presque toutes les localités du Département (Komena, 2019). Ce brassage des ethnies et des nationalités a permis de recenser un nombre élevé de plantes comestibles et des habitudes alimentaires différentes, en complément aux savoir-faire des autochtones Gouro. Les femmes ont été plus rencontrées au cours des enquêtes (64,69 \%), révélant ainsi que les plantes comestibles sauvages constituent un secteur en grande partie aux mains des femmes rurales. De plus, avec 85 \% d'enquêtées ayant entre 15 et 50 ans, elles représentent une tranche active de la population locale, et savent lire et écrire dans $60 \%$ de cas. Ce qui est un atout précieux pour la valorisation des plantes comestibles sauvages visant un impact social rapide sur des ménages vulnérables (AGRIDAPE, 2016 ; HLPE, 2018).

Le nombre cumulé d'espèces spontanées utiles recensées en Côte d'Ivoire, depuis les travaux de Portères (1935) jusqu'à ce jour, est de 715, dont 553 plantes médicinales, 238 plantes comestibles et 219 plantes artisanales (Piba, 2016 ; Ouattara et al., 2016). L’inventaire réalisé à Zuénoula (76 espèces) a permis d'identifier 31,93 \% d'espèces comestibles déjà recensées. Cette diversité peut s'expliquer par la phytogéographie particulière de la région, car le Département appartient à la zone végétative de transition savanes-forêts, ce qui a sans doute favorisé le peuplement d'un nombre important de taxons (Bouquet \& Debray, 1974).

Dans le Département de Bondoukou, appartenant au secteur de transition savanes-forêts du Nord-est de la Côte d'Ivoire, 81 espèces comestibles ont été répertoriées (Ouattara et al., 2016). On remarque que 39 espèces recensées à Zuénoula ne sont pas signalées à Bondoukou. Parmi elles, on peut citer Abrus precatorius et Costus afer, deux plantes pourtant inventoriées dans la flore médicinale locale du Gontougo (Béné et al., 2016). Toujours au niveau du 
secteur de transition savanes-forêts, dans le Département Séguéla (Nord-ouest de la Côte d'Ivoire), Ambé (2001) a recensé 75 espèces fruitières sauvages utilisées par une population locale Malinké. On remarque que seulement 26 espèces sont communes et 14 espèces recensées dans notre étude n’ont pas été signalées par cet auteur. Parmi elles, on peut citer Borassus aethiopum, Capsicum frutescens, Sesamum radiatum, Solanum indicum, des plantes pourtant bien répandues dans la végétation des savanes et des forêts au Nord du pays (Aké-Assi, 1984 ; 2001). On peut donc affirmer que les systèmes alimentaires des peuples enquêtés à Zuénoula, Bondoukou et Séguéla, bien que s'appuyant sur une même végétation de transition savanes-forêts, sont traditionnellement différents.

Dans une autre étude consacrée aux plantes alimentaires spontanées du Département de Gagnoa (Centre-Ouest de la Côte d'Ivoire), secteur Soudanoguinéen autrefois caractérisé par la forêt dense humide semi-décidue, Kouamé et al. (2008) ont inventorié 72 espèces. On constate que 46 espèces recensées à Zuénoula ne sont pas citées par ces auteurs. C’est le cas du Rônier (Borassus aethiopum) et du Néré (Parkia biglobosa) deux espèces exclusivement savanicoles et bien connues en Côte d'Ivoire. De même, certaines espèces comme Garcinia kola et Beilschmiedia mannii, arbres des forêts denses humides, signalés à Gagnoa, ne figurent pas sur la liste de la présente étude. Ceci montre que l'alimentation traditionnelle des populations indigènes est influencée par la biodiversité locale.

Au Sud de la Côte d’Ivoire, chez le peuple Krobrou (Département d'Agboville), un recensement plus étendu au Département a rapporté 96 plantes reparties en 87 genres et 48 familles (Bédiakon et al., 2018). Par comparaison, 52 espèces figurant sur notre liste n’ont pas été citées par le peuple Krobrou. On constate néanmoins que Borassus aethiopum, « sentinelle de savanes » a été signalée dans la flore locale d'Agboville, qui est pourtant une zone de forêt dense humide au Sud du pays. Ce qui indique, selon Soro et al. (2018), que le paysage forestier ivoirien est en pleine transformation. Enfin, 54 espèces soit 71,05\%, sont très connues. Ce rapport est bien plus élevé que ceux de Bondoukou (54,14 \%) et de Gagnoa (9, 72 \%) (Kouamé et al., 2008 ; Ouattara et al., 2016). Les populations rurales de Zuénoula ont donc une bonne connaissance de la flore comestible locale. L'ensemble des résultats évoqués montre que le nombre d'espèces spontanées consommées varie en fonction des groupes ethniques locales et les sites étudiés. Ce constat est largement partagé par tous les auteurs.

En outre, la présente étude a révélé la consommation de Gymnema sylvestre et de Bixa orellana en Côte d'Ivoire. En effet, G. Sylvestre, bien qu'elle soit répandue dans la flore ivoirienne sous forme de buissons lianescents dans des savanes boisées et des forêts denses (Aké-Assi, 2001), n'a pas d'utilités connues en Côte d'Ivoire, ni dans la pharmacopée ivoirienne, 
ni dans les systèmes alimentaires et nutritionnels traditionnels. C'est auprès d'une communauté Burkinabé du village de Dhèzra (sous-préfecture de Kanzra) que G. sylvestre a été présentée pour la première fois comme aliment dans nos enquêtes. Le mode de préparation est classique : les feuilles récoltées à l'état frais sont bouillies, puis transformées en pâte. Celle-ci, assaisonnée ou non, est consommée directement ou utilisée comme plat d'accompagnement. Le plus souvent, la pâte est ajoutée aux sauces ou aux autres aliments durant la cuisson. En pharmacopée locale, ces communautés utilisent les tiges et parfois les feuilles de cette espèce pour soigner les plaies, les morsures d'insectes et de serpents, et pour obtenir la cicatrisation rapide des blessures. Dans certains pays d'Afrique et d'Asie, précisément en Inde, la plante est intensément étudiée et exploitée pour produire des compléments alimentaires et des médicaments à actions diverses : digestives, purgatives, hypotensives, antidiabétiques, antimicrobiennes, anti-inflammatoires, antigrippales, pertes de poids, baisses de cholestérols, etc. (Jiofack et al., 2010 ; Hajare, 2018). Vu son potentiel économique et social énorme, et face à la déforestation rapide et aux pillages des ressources médicinales locales de grand intérêt, un programme de valorisation durable de G. sylvestre doit être urgemment mis en place en Côte d'Ivoire.

Quant à Bixa orellana (le Roucou), il n'existe à notre connaissance aucune étude botanique réalisée en Côte d'Ivoire l'ayant signalée comme une plante comestible. Le Roucou est plutôt exploité dans la décoration et le cosmétique (Kwassi et al., 2019). Par contre à Zuénoula, les graines de $B$. orrellana sont utilisées comme colorants de certains bouillons alimentaires (piments, poissons, crevettes, etc.). Sous cette forme, le Roucou est bien consommé en Asie, et son usage local ou industriel dans l'alimentation humaine est suffisamment documenté (Hagiwara et al., 2002). En plus, selon ces auteurs, la Bixine, principal complexe colorant des graines de Roucou, possède des propriétés thérapeutiques et nutritionnelles diverses: antibactériennes, antiparasitaires, anti-inflammatoires, diurétiques, hypotensives, laxatives, digestives, etc. Nous suggérons d'effectuer une enquête diagnostique régionale dans le but d'évaluer les caractéristiques alimentaires et nutritionnelles du Roucou ivoirien, et prévenir ainsi des risques éventuels d'intoxication.

Les organes fruitiers $(47,87 \%)$ et les feuilles $(30,85 \%)$ ont été inventoriés dans les mêmes proportions (test $U=1,74)$. Réunis (78,72 \%), ils occupent une part significative dans le pool des organes traditionnellement consommés par la population (Chi $2=72,02$ ). Ce qui présente un avantage pour les paysans ruraux car, la consommation régulière des feuilles et fruits procure à l'organisme des micronutriments essentiels, sains et bon marché. Les feuilles sont généralement exploitées par des populations locales pour des repas familiaux, contrairement aux fruits souvent consommés sur les lieux 
même des cueillettes. Parmi les 29 espèces de légumes-feuilles, 10 espèces ne figurent pas sur les listes des trois Régions déjà évoquées (Bondoukou, Gagnoa, Agboville). Ce sont: Abrus precatorius, Albizia zygia, Annona senegalensis, Celosia trigyna, Corchorus aestuans, Corchorus tridens, Gymnema sylvestre, Justicia galeopsis, Lonchocarpus cyanescens, Phyllanthus reticulatus. Les espèce $A$. precatorius et $S$. mombin sont des plantes médicinales citées par des auteurs contre les diarrhées (Béné et al., 2016 ; Gnagne et al., 2017), mais à Zuénoula, leurs feuilles sont aussi consommées par les populations locales, le plus souvent sur le site du prélèvement. Lors de la mastication, les feuilles crues de $A$. precatorius donnent un goût sucré caractéristique dont les consommateurs sont friands. Quant à S. mombin, les feuilles sont emballées puis bouillies au feu doux avant d'être consommées. Ces formes d'utilisation locales de ces deux feuilles donnent l'avantage aux consommateurs de se protéger naturellement contre les maladies gastro-intestinales (Bouquet \& Debray, 1974).

Dans la nature, G. sylvestre forme des friches buissonnantes vivaces fortement enracinées, ce qui rend le travail du sol très pénible, selon les cultivateurs rencontrés. Quant à $C$. trigyna, elle constitue des adventices redoutables dans les champs et les jachères pendant les saisons pluvieuses. Pour freiner leur profusion, les paysans font aujourd'hui usages excessifs d'herbicides. Ces méthodes de défrichages, qui ont presque éteint la cucurbite Telfairia occidentalis, menacent aujourd'hui la survie de nombreuses espèces utiles comme Glyphaea brevis. C'est un triste constat car cette dernière est la seule espèce à produire trois organes utilisés pour des repas familiaux (feuilles, fleurs, fruits).

\section{Conclusion}

La recherche des plantes sauvages à usages alimentaires en vue de lutter contre la faim et la pauvreté en milieu rural a conduit à réaliser une étude ethnobotanique dans la flore locale du Département de Zuénoula. Elle a permis de recenser 76 espèces, composées de 47,87 \% de plantes fruitières et 30,85 $\%$ de légumes-feuilles. A l'échelle nationale, la flore locale contient 31,93 \% d'espèces comestibles poussant dans les plantations, les jachères, les savanes et les forêts. Les arbres et les herbes fournissent 66,48 \% d'organes réellement utilisés, les arbustes et les lianes 33,52 \%. Par rapport aux régions déjà enquêtées, Agboville, Bondoukou, Gagnoa et Séguéla, les plantes sauvages alimentaires sont plus connues et plus consommées à Zuénoula. Les femmes en sont les plus utilisatrices. Certaines espèces sont produites dans des potagers et vendues sur des marchés locaux: Cleome gynandra, Sesamum indicum, Solanum indicum, Talinum triangulare. D'autres, par contre, sont en voie d'extinction et avec elles des savoir-faire traditionnels. Ce sont Adansonia digitata, Glyphaea brevis, Irvingia gabonensis, Telfairia 
occidentalis. Enfin, deux espèces peu citées dans la flore ivoirienne comme aliments ont été recensées : Bixa orellana et Gymnema sylvestre. Il n'existe presque plus de forêts naturelles à Zuénoula, plutôt des jachères et/ou des fragments de forêts anciennes. Au regard du potentiel nutritionnel et rustique de ces plantes sauvages, et face aux enjeux socio-économiques, environnementaux et climatiques pressants, la valorisation agronomique des espèces indigènes à usages familiaux est nécessaire et prioritaire. Elle contribuera à renforcer l'accessibilité aux aliments locaux et à accroître la qualité et la diversité des régimes alimentaires dans les zones rurales.

\section{References:}

1. Adjanohoun, E., \& Aké-Assi, L. (1979). Contribution au recensement des plantes médicinales de Côte d'Ivoire. Centre National de Floristique, Université d'Abidjan, 358 p.

2. Agridape (2016). Revaloriser les espèces agricoles traditionnelles sous-utilisées. Edition Afrique Francophone, 32 (2) : 32 p.

3. Aké-Assi, L. (1984). Flore de la Côte-d'Ivoire : étude descriptive et biogéographique, avec quelques notes ethnobotaniques, Thèse de Doctorat d'Etat, Université d'Abidjan, F.A.S.T., Nº d'ordre : 008/84, $1206 \mathrm{p}$.

4. Aké-Assi, L. (2001). Flore de la Côte d’Ivoire : Catalogue Systématique, Biogéographie et écologie. II. Boissiera, 57 : 1-396.

5. Ambé, G.A. (2001). Les fruits sauvages comestibles des savanes guinéennes de la Côte d'Ivoire : état de la connaissance par une population locale, les Malinkés. Biotechnologie, Agronomie, Société \& Environnement, 5 (1) : 43-58.

6. Ambé, G.A. (2003). Etude ethnobotanique et identification de quelques espèces végétales sauvages prometteuses : cas des Malinkés du Département de Séguéla (Côte d'Ivoire). Thèse de Doctorat, Fac. Univ. Sc. Agron. Gembloux, 203 p.

7. APG IV (2016). An update of the Angiosperm Phylogeny Group classification for the orders and families of flowering plants. Botanical Journal of the Linnean Society, $161: 1-20$.

8. Banque mondiale (2018). Pour que demain ne meure jamais. La Côte d'ivoire face au changement climatique, $64 \mathrm{p}$.

9. Bédiakon, B.K.D., Beugré, G.A.M., Yao, K., \& Ouattara, D. (2018). Enquête ethnobotanique sur les plantes spontanées alimentaires dans le département d'Agboville (Côte d'Ivoire). International Journal of Scientific \& Engineering Research 9 (11) : 1843-1856.

10. Béné, K., Camara, D., Fofie, N., Bra, Y., Kanga, Y., Yapi, A.B., Yapo, Y.C., Ambé, S.A., \& Zirihi, G.N. (2016). Étude ethnobotanique des plantes médicinales utilisées dans le Département de Transua, District 
du Zanzan (Côte d'Ivoire). Journal of Animal \& Plant Sciences 27 (2): 4230-4250.

11. Bouquet, A., \& Debray, M. (1974). Plantes médicinales de la Côte d'Ivoire. Revue OSTORM, Paris. 231 p.

12. Dajoz (1982). Précis d'écologie. Ecologie fondamentale et appliquée Ed. Gauthier-Villers paris 503 pp.

13. Ducroquet, H., Tillie, P., Louhichi, K., \& Gomez, Y.P.S. (2017). L'agriculture de la Côte d'Ivoire à la loupe, état des lieux des filières de production végétales et animales et revue des politiques agricoles. JRC Science for Policy Report. Publications Office of the European Union, Luxembourg, 244 p.

14. Gautier-Béguin, D. (1992). Étude ethnobotanique des plantes de cueillette à utilisation alimentaire dans un village au sud du V -Baoulé (Côte d'Ivoire). Thèse de Doctorat. Université de Genève, Laboratoire de Botanique systématique et floristique, $382 \mathrm{p}$.

15. Gnagne, A.S., Camara, D., Fofié, N’Guessan, B.Y., Béné, K., \& Zirihi, G.N. (2017). Étude ethnobotanique des plantes médicinales utilisées dans le traitement du diabète dans le Département de Zouénoula (Côte d'Ivoire). Journal of Applied Biosciences 113 : 11257-11266.

16. Hagiwara, A., Imai N., Ichihara, T., Sano, M., Tamano, S., Aoki, H., Yasuhara, K., Koda, T., Nakamura, M., \& Shirai, T. (2002). A thirteenweek oral toxicity study of annatto extract (norbixin), a natural food color extracted from the seed coat of annatto (Bixa orellana), in Sprague-Dawley rats. Food Chemical Toxicology 41 (8): 1157-64.

17. Hajare, R. (2018). Comparing Modified and Relationship Study of Gymnema Sylvestre against Diabetes. Journal of Global Diabetes \& Clinical Metabolism. India, 3 (1) : 1-3.

18. Hlpe (2018). Nutrition et systèmes alimentaires. Rapport du Groupe d'experts de haut niveau sur la sécurité alimentaire et la nutrition du Comité de la sécurité alimentaire mondiale, Rome. 12 : 190 p.

19. Jiofack, T., Fokunang, C., Guedje, N., Kemeuze, V., Fongnzossie, E., Nkongmeneck, B.A., Mapongmetsem, P.M., \& Tsabang, N. (2010). Ethnobotanical uses of medicinals plants of two ethnoecological regions of Cameroon-International Journal of Medicine and Medical Sciences, 2(3): 60-79.

20. Komena, B. (2019). Inégalités, pauvreté et soutenabilité des ressources naturelles dans l'Ouest de la Côte d'Ivoire. Éthique et économique, 16 (2) :12 p.

21. Koné, M. (2015). Évolution du couvert forestier dense et impact de la déforestation sur la migration de la boucle du cacao en Côte d'Ivoire. Thèse de Doctorat, Université Nangui Abrogoua, UFR Sciences de la Nature, 185 p. 
22. Kouamé, N.M-T. (2000). Contribution à l'étude des plantes spontanées alimentaires du département d'Oumé (Côte d'Ivoire). Mémoire de D.E.A d'Ecologie tropicale (Option : Végétale). Université de Cocody Abidjan, Côte d'Ivoire, 122 p.

23. Kouamé, N.M.T., Gnahoua, G.M, Kouassi, K.E, \& Traoré, D. (2008). Plantes alimentaires spontanées de la région du Fromager (CentreOuest de la Côte d'Ivoire) : flore, habitats et organes consommés. Sciences \& Nature 5 (1) : 61-70.

24. Kwassi, D.S.A., Kouassi, A.F., \& Aké-Assi, E. (2019). Diversité floristique des plantes a potentialité décorative issues des formations naturelles du Nord de la Côte d'Ivoire. European Scientific Journal,15: 1857-7881.

25. N’Da, D.H., Adou, Y.C.Y., N’Guessan, K.E., Koné, M., \& Sagne, Y.C. (2008). Analyse de la diversité floristique du parc national de la Marahoué, Centre Ouest de la Côte d'Ivoire. Afrique Science, 4 (3) : 552-579.

26. N’Guessan, K., Kouamé, N.M-T., Assi-Kaudjhis, C., \& Aké, C.B. (2015). Ethnobotanical study of spontaneous wild plants used for food by krobrou People, in the South of Côte d'Ivoire, 4 (2) : 1354-1365.

27. N'Dri, P. (1986). Contribution à l'étude de quelques plantes alimentaires spontanées de la Région de Divo (Côte d'Ivoire). Mémoire de D.E.A. d'écologie tropicale, Université de Cocody, Abidjan, Côte d'Ivoire, 65 p.

28. Ouattara, N.D., Gaille, E., Stauffer, F.W., \& Bakayoko, A. (2016). Diversité floristique et ethnobotanique des plantes sauvages comestibles dans le Département de Bondoukou (Nord- Est de la Côte d'Ivoire). Journal of Applied Biosciences, 98 : 9284 - 9300

29. PDI (2003). Plan Directeur d'Irrigation en Côte d'Ivoire, 2002-2004.

30. Piba, S.C. (2016). Diversité floristique et potentiel en espèces sources de produits forestiers non ligneux de la forêt classée de Yapo-Abbe : contribution pour un aménagement durable. Thèse de doctorat, Université Nangui Abrogoua, UFR des Sciences de la Nature, Abidjan (Côte d'Ivoire), $253 \mathrm{p}$.

31. PNUD (2015). Rapport national sur le développement humain en Côte d'Ivoire. Cohésion sociale et reconstruction du pays, $191 \mathrm{p}$.

32. Portères, R. (1935). Plantes toxiques utilisées par les peuplades Dan et Guéré de la Côte d'Ivoire. Bulletin du comité d'Études Historiques et Scientifiques de l'Afrique Occidentale Française, 13 : 128-140.

33. RGPH (2014). Recensement Général de la Population et de l'Habitat, Rapport d'exécution et présentation des résultats, Côte d'Ivoire 49 p. 
34. Sodefor (1996). Plan d'aménagement de la forêt classée de Bouaflé. Ministère de l'agriculture et des ressources animales, Côte d'Ivoire, 61 p.

35. Soro, Y., N’Dri, A.B., Bakayoko, A., \& Gignoux, J.G. (2018). Analyse de la végétation dans un écotone forêt-savane d'Afrique de l'Ouest dans un contexte de boisement de savanes. Revue de l'Environnement et de la Biodiversité, $3: 54-72$.

36. Yao, N.R., Oulé, A.F., \& N’Goran, K.D. (2013). Etude de vulnérabilité du secteur agricole face aux changements climatiques en Côte d'Ivoire, Rapport final. Ministère de l'Environnement et du Développement durable, Service de suivi des programmes nationaux, Côte d'Ivoire. $105 \mathrm{p}$. 